\title{
Bienestar infantil y capacidades humanas entre niños, niñas y adolescentes (NNA) trabajadores de zonas agrícolas rurales en México
}

\section{Welfare and human capabilities among children and adolescents workers of rural agricultural areas in Mexico}

\author{
Francisco Pérez-Soto, Pablo Emilio Escamilla-García, Esther Figueroa \\ Hernández, Rebeca Pérez-Figueroa \\ División de Ciencias Económico Administrativas de la Universidad \\ Autónoma Chapingo, México \\ Instituto Politécnico Nacional, México \\ Centro Universitario UAEM Texcoco, Universidad Autónoma del Estado de \\ México, México
}

\section{Resumen}

El propósito de esta investigación fue examinar el contexto del trabajo infantil llevado a cabo por hijos de trabajadores agrícolas migrantes en México. El estudio encuestó a 558 niños, niñas y adolescentes (NNA) pertenecientes a familias agrícolas migrantes. El instrumento de recopilación de información fue basado en el Parental Acceptance-Rejection Questionnaire (PARQ) y el Youth Self-Report (YSR), alineando las dimensiones de análisis a las capacidades humanas centrales propuestas por Nussbann. Los resultados mostraron elementos críticos que afectan el bienestar infantil, y la vulnerabilidad en diferentes capacidades humanas que condicionan el desarrollo humano de los NNA en zonas rurales.

Palabras clave: Trabajo infantil, bienestar infantil, capacidades humanas, jornaleros migrantes agrícolas.

\section{Abstract}

The purpose of this research was to examine the context of child labor carried out by children from migrant agricultural laborers in Mexico. The study applied a methodology based on the identification of current migrant agricultural laborer according to the main crops cultivated in each region, thus children were located within migrant agricultural families. 558 children were surveyed by conducting individual in-depth interviews with working children and applying a multi-category questionnaire aligned with the central human capabilities of Nussbann and considering the Parental Acceptance-Rejection Questionnaire (PARQ) model and the Youth Self-Report (YSR). Results showed critical elements than compromise child welfare, it was noted a significant vulnerability of the rights of the children and an adverse environment which lead children to an improper growth limiting their human capacities for a personal development.

Key words: Child labor, child welfare, human capabilities, agricultural migrant laborers. 


\section{INTRODUCCIÓN}

\section{Contexto del trabajo infantil}

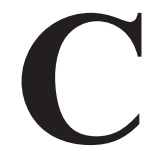

ada año, aproximadamente 300 mil niños, niñas y adolescentes (NNA) salen de sus lugares de origen para emigrar con sus familias a otras regiones de México en busca de trabajo e ingresos. La mayoría de los hijos de jornaleros agrícolas provienen de comunidades rurales indígenas, por lo que la migración representa para ellos cambios en sus costumbres, cultura e idioma. Estos NNA suspenden sus estudios debido a complicaciones administrativas surgidas al cambiar su lugar de residencia, lo que los orilla a trabajar en los campos agrícolas (Ramírez-Romero et al., 2006: 43).

Las cifras oficiales reportadas por el Instituto Nacional de Estadística y Geografía (INEGI) muestran que, en México, hay 3.6 millones NNA menores de 17 años que trabajan, de los cuales 1.1 millones tienen menos de 14 años, esto ubica a México en el sitio 49 en tasa de trabajo infantil de entre cinco y 14 años (INEGI, 2019). El problema del trabajo infantil debe abordarse, en primer lugar, no solo al enfatizar las violaciones de las leyes internacionales actuales, sino principalmente al analizar los diferentes aspectos y situaciones que originan el problema. Las causas de origen y existencia del trabajo infantil pueden variar según el contexto de cada lugar, sin embargo, la pobreza y las imperfecciones de las economías pueden llevar a la proliferación de estos problemas (Baland y Robinson, 2000: 663). Los factores socioeconómicos y políticos, especialmente el desempleo y la migración, juegan un papel importante en el trabajo infantil (Mert y Kadioglu, 2014: 171). Cavalcanti (2003: 741) argumenta que el trabajo infantil en los países en desarrollo resulta de la limitada oferta de sistemas educativos, y sobre todo de la calidad del sistema en cuestión. De esta manera, padres y/o tutores de los NNA infieren un valor nulo en la educación, obligando así al infante a trabajar. Tang et al. (2016: 1) mencionan que es más probable que un niño que vive en un área rural trabaje en contraste con NNA de hogares en zonas urbanas. Los modelos de roles también pueden llevar al trabajo infantil, los NNA que realizan tareas productivas en un contexto social determinado pueden ser susceptibles de imitación por otros NNA que viven en el mismo entorno (Alacahan, 2010: 133).

El trabajo infantil en las actividades agrícolas es una realidad en los países en desarrollo. Se han llevado a cabo investigaciones para analizar los efectos negativos en el rendimiento académico y la deserción escolar 
(He, 2016: 130; Beuermann, 2015: 988; Dumas, 2012: 773; Debebe, 2010: 25). También se han estudiado los efectos adversos sobre la salud de los NNA (Thabet et al., 2010: 89; O'Donnell et al., 2005: 437). Igualmente hay estudios sobre los impactos financieros en las economías familiares y domésticas (Bouoiyour y Miftah, 2014: 75; De Carvalho, 2012: 399; Ferguson, 2005: 101). La investigación teórica y empírica sobre el trabajo infantil es amplia, sin embargo, los estudios específicos sobre el trabajo infantil en México, particularmente en el sector agrícola, son limitados. Miranda (2015: 151) caracterizó el trabajo infantil rural en México encontrando que existe una propensión similar al trabajo infantil independiente del sexo. Por otra parte, Monroy y Juan (2009: 12) analizaron la migración campesina para comprender la incorporación del trabajo infantil en regiones indígenas de México encontrando que la existencia del trabajo infantil tiene que ver la supervivencia familiar. Aunque estos estudios son valiosas aportaciones al tema del trabajo agrícola infantil en México en su mayoría se centran en las consecuencias políticas / sociales / económicas. Por lo que aún no se han explorado las consecuencias para el individuo y cómo esto afecta su desarrollo personal en términos de bienestar y desarrollo social en las zonas rurales donde habitan los jornaleros agrícolas. Beegle et al. (2009: 80) mencionan que el trabajo infantil en hogares agrícolas en países en desarrollo tiene consecuencias negativas en el desarrollo a largo plazo de los NNA. Por lo tanto, el caso del trabajo infantil en el sector agrícola de México puede enfocarse desde el punto de vista del bienestar vinculado a las capacidades humanas que determinan un desarrollo personal.

\section{Capacidades humanas y bienestar infantil}

El bienestar infantil debe analizarse primero a partir de las teorías de las necesidades humanas básicas sin las cuales un ser humano no puede considerarse satisfecho en su desarrollo ni se le pueden atribuir el concepto de dignidad humana (Monereo-Atienza, 2005: 265). Al analizar las capacidades humanas del bienestar, se destacan dos teorías, el Enfoque de capacidad de Amartya Sen y las Capacidades humanas centrales de Martha Nussbaum. Amartya Sen propone un enfoque de capacidad que proporciona una crítica formal y precisa de la economía del bienestar. Martha Nussbaum ofrece una ética de desarrollo de las capacidades basada en la tradición aristotélica (Gasper, 1997: 281). La teoría de Sen no emplea un umbral o una lista específica de capacidades ni hace un uso teórico central del concepto de dignidad humana (Nussbaum, 2011: 89). Por otro lado, la teoría de Martha Nussbaum establece la defensa de un "ser humano" con capacidades y ne- 
cesidades comunes a todos, independientemente de su género, clase, raza o nacionalidad. Las capacidades son aquellas libertades sustanciales que una persona debe disfrutar para lograr la vida que su visión o razón le permite valorar (Sen, 1999: 32). Por lo tanto, para analizar las implicaciones que tiene el trabajo infantil en los NNA, se puede aplicar la identificación de capacidades. Las capacidades deben estar vinculadas a la noción de la dignidad humana y la forma de vivir una vida digna. Sin embargo, una sociedad que no garantiza los niveles mínimos de cada capacidad, para resolverlos sobre principios políticos y garantías constitucionales, se aleja de la posibilidad de lograr la justicia para todos (Nussbaum, 1997: 273). Las capacidades humanas centrales son (Nussbaum, 2000: 33): 1. La vida. 2. Salud corporal. 3. Integridad corporal. 4. Sentidos, imaginación y pensamiento. 5. Emociones. 6. Razón práctica. 7. Afiliación: A) Poder vivir con y hacia los demás; B) Tener las bases sociales de respeto propio y no humillación. 8. Otras especies. 9. Recreación. 10. Control sobre el propio entorno: A) Político; B) Material.

\section{Las capacidades humanas en los programas sociales de México}

Durante varios años, en México, la perspectiva de las capacidades se ha asumido como un marco teórico para el desarrollo de programas y políticas públicas. Los programas sociales han buscado vincular tres elementos que se perciben como sustanciales para fortalecer las capacidades de las personas en situación de pobreza (alimentos, recursos y activos) a través de la distribución de servicios y apoyos relacionados con la salud, educación y alimentos. Sin embargo, estos programas han sido parciales en su definición y uso como red social y como marco para analizar la complejidad del desarrollo humano, particularmente en los NNA de zonas rurales. Por lo tanto, esta investigación tiene como objetivo analizar las percepciones de los NNA hijos de trabajadores migrantes agrícolas en México para determinar la vulnerabilidad a la que están expuestos en función de las capacidades centrales propuestas por Nussbaum. Con esto se busca responder a la pregunta ¿En qué grado se vulneran las capacidades centrales humanas de los NNA que laborar en los campos agrícolas de México?

\section{Materiales Y MÉTODOS}

\section{La ubicación del estudio y los participantes}

La investigación se realizó durante la temporada de cosecha agrícola en los estados de Veracruz, Oaxaca y Sinaloa en México. Los participantes 
del estudio eran hijos de jornaleros agrícolas migrantes. Estos jornaleros y toda su familia (incluidos los NNA) abandonan sus lugares de origen para trasladarse a la región o estado en que se encuentran las tierras cultivadas, cuando termina la temporada agrícola, las familias regresan a sus lugares de origen o buscan nuevas regiones con tierras de cultivo activas.

Dado que los NNA encuestados formaban parte de las familias de migrantes agrícolas, la metodología inicial se centró en la localización de los jornaleros. Los trabajadores agrícolas migrantes fueron seleccionados de acuerdo con el siguiente método: dada la naturaleza de los procesos de producción agrícola, la cosecha es la etapa en la que se contrata a la mayoría de los jornaleros, por lo que el estudio se centró en localizar trabajadores agrícolas durante esta etapa. Con estos antecedentes, para estimar el número de jornaleros agrícolas en México se consideraron tres variables principales: i) mano de obra necesaria para la etapa de la cosecha por cultivo; ii) superficie cosechada de los cultivos; iii) nivel tecnológico (utilización de tecnología que sustituye mano de obra).

Dada la disponibilidad de información, solo es posible obtener las primeras variables, con las cuales se estima un universo de muestreo bajo la siguiente metodología: i) se obtuvo la cantidad de superficie cosechada anualmente de cada cultivo a nivel nacional en hectáreas. ii) Se consultó la estructura de costos de los principales cultivos conforme al SIAP (Sistema de Información Agroalimentaria y Pesquera) y a los Comités Sistema Producto Nacionales y se obtuvo la mano de obra promedio empleada en la etapa de la cosecha de cada cultivo. iii) Se estimó el total de mano de obra empleada anual por cultivo a nivel nacional con el producto de la superficie cosechada y la mano de obra ocupada por unidad de superficie. El procedimiento matemático se describe a continuación:

$$
\text { MOTE }=\sum\left(S C_{i} * M O C_{i}\right)
$$

Donde:

MOTE: Mano de Obra Anual Total Estimada.

$S C_{i}$ : Superficie cosechada anual nacional del cultivo $i$.

$M O C_{i}$ : Mano de Obra utilizada por unidad de superficie (ha) en la cosecha en el cultivo $i$.

Para determinar la cantidad de mano de obra utilizada conforme a cada cultivo $\left(\mathrm{MOC}_{\mathrm{i}}\right)$ se utilizó información del SIAP referente a los cultivos que 
representan 98 por ciento de la superficie sembrada nacional (41 cultivos). El cálculo se realizó como sigue:

$$
M O C_{i}=\left(\frac{N h_{c}}{K}\right) * C
$$

Donde:

$M O C_{i}$ : Mano de Obra utilizada por unidad de superficie (ha) en la cosecha en el cultivo $i$.

$N h_{c}:$ Número de horas ocupadas por corte en el cultivo.

$C$ : Número de cortes del cultivo.

Con el anterior procedimiento se determinó que el universo de muestreo se concentrara en los cultivos que representan 90 por ciento de jornales utilizados en la cosecha de las 41 especies analizadas lo cual corresponde a los siguientes 12 cultivos: caña de azúcar, tomate rojo, café cereza, naranja, Mango, manzana, calabacín, tomate verde, chile verde, cantalupo, uva, melocotón.

Una vez que se identificaron los cultivos, se seleccionaron tres estados, Veracruz, Oaxaca y Sinaloa, regiones principales donde se siembran los cultivos identificados. Con base en la información de las agencias locales de agricultura, fue posible establecer una población promedio de 3,500 trabajadores agrícolas migrantes en los tres estados y que actualmente estaban trabajando en ese momento. Con una población de 3,500, una confianza de 95 por ciento $(Z \alpha=1.96)$, margen de error $=5$ por ciento y una proporción de muestra $=50$ por ciento el tamaño de la muestra resultó en 198. Debe señalarse que el valor de 198 representa la cantidad total de trabajadores agrícolas migrantes (padres). Esto es, el número de familias solamente, el número final de NNA integrantes de dichas familias fue conocido al momento de interactuar con los padres y se identificaron 558 NNA. Es importante señalar que el procedimiento aplicado obedece a la carencia total de censos estadísticos de NNA trabajadores en campos agrícolas de zonas rurales.

\section{El instrumento de investigación}

Se elaboró un cuestionario con dos variables principales: características del entorno y aspectos físicos y mentales del niño. Estas variables se desglosaron en las siguientes dimensiones: aspectos demográficos, educación, composición/organización familiar, actividades recreativas, trabajo en el 
sector agrícola, condición física y salud, conducta emocional y percepción de derechos. Dichas categorías se definieron a fin de que los resultados se alinearan con las capacidades humanas centrales propuestas por Nussbaum. La construcción y aplicación del cuestionario se basó en dos metodologías. Se utilizó el modelo del Parental Acceptance-Rejection Questionnaire (PARQ) para evaluar el posible trastorno de la conducta en los NNA que trabajan. Este modelo se basa en la teoría de aceptación y rechazo de los padres desarrollada por Rohner (1987: 26) que es una teoría de la socialización que intenta predecir el ajuste psicológico de los NNA independientemente de las diferencias culturales, étnicas, raciales, de género, socioeconómicas u otras. A los efectos del estudio, este modelo se utilizó para medir los niveles de calidez, afecto, hostilidad, agresión e ira en el comportamiento de los NNA cuando interactúan con otros miembros de la familia y con su entorno. Adicionalmente el cuestionario replicó elementos del Youth Self-Report (YSR), que es un autoinforme diseñado por Achenbach (1991: 175) para obtener información sistematizada directamente de NNA. El modelo YSR se utilizó con un enfoque en las percepciones y opiniones de los NNA en términos de actividades laborales, de ocio y de atención médica. El cuestionario final se integró de 90 reactivos en escala de Likert.

\section{Recopilación de la información}

El proceso de recopilación de información se realizó mediante sesiones con grupos de tres a cinco NNA por sesión, conducidas por profesionales en psicología infantil y bajo la observación de los padres/tutores. Se aplicó el método de terapia de juegos participante y no directiva a fin de la obtención de información se diera de forma natural al interactuar con el menor. La terapia de juegos igualmente permitió analizar la conducta del niño mediante actividades lúdicas para obtener la información de cada categoría del cuestionario. Cabe aclarar que los reactivos de Likert del cuestionario fueron llenados por los psicólogos en función de lo observado en las sesiones y no por los NNA y/o padres/tutores, esto debido a que el cuestionario era traducido por los especialistas a un lenguaje entendible para cada menor en función de su rango de edad. Inicialmente se realizó una prueba piloto para evaluar las terapias de juegos y el cuestionario con solo 25 NNA. Los resultaron mostraron un comportamiento adecuado en términos estadísticos por lo que solo se modificó el orden de las actividades. Las sesiones de la terapia se realizaron en un tiempo promedio de 30 a 45 minutos, con ocho sesiones simultaneas por día. 


\section{Validación y confiabilidad del instrumento}

Se condujeron las pruebas de fiabilidad y validez aplicando el análisis de varianzas individuales y totales por cada respuesta en las diferentes dimensiones del cuestionario. Se medió la confiabilidad del instrumento mediante el coeficiente de Cronbach (ver Tabla 1) en donde se obtuvieron valores dentro del rango adecuado.

Tabla 1: Resultados de Alpha de Cronbach

\begin{tabular}{llc}
\hline Variables & \multicolumn{1}{c}{ Dimensión } & Alpha de Cronbach \\
\hline Características & Aspectos demográficos & 0.92 \\
del entorno & Composición / organización familiar & 0.84 \\
& Trabajo en el sector agrícola & 0.80 \\
& Actividades recreativas & 0.82 \\
Aspectos físicos y & Educación & 0.81 \\
mentales del niño & Condición física y salud & 0.80 \\
& $\begin{array}{l}\text { Conducta emocional y percepción de } \\
\text { derechos }\end{array}$ & 0.84 \\
\hline
\end{tabular}

Fuente: elaboración propia.

Posteriormente cada dimensión de análisis fue validada mediante un análisis de correlaciones, los resultados obtenidos se muestran en la Tabla 2.

Se estableció que para el análisis solo se observaron cinco correlaciones negativas contra setenta y nueve correlaciones positivas. Lo que implica una confiabilidad adecuada para las relaciones entre las dimensiones de análisis establecidas. En lo que respecta a análisis factorial confirmatorio la medida de Kaiser-Meyer-Olkin de adecuación de muestreo resultó en 0.716 y la prueba de esfericidad de Bartlett en 48.417. Esto muestra que los datos son confiables para las pruebas realizadas.

\section{Consideraciones éticas}

De acuerdo con los principios éticos formulados por la American Psychological Association (APA) en su enmienda 2010 (APA, 2010) así como a las recomendaciones plasmadas en el Documento III-02 del MPA PDD de la Organización Mundial del Trabajo sobre las consideraciones éticas para la investigación sobre NNA sometidos a trabajo infantil (OIT, 2003). 
Bienestar infantil y capacidades humanas entre niños, niñas y adolescentes (NNA) ... / F. PÉREZ SOTO et al.

Tabla 2: Correlaciones entre dimensiones por coeficiente de Pearson

\begin{tabular}{|c|c|c|c|c|c|c|c|c|}
\hline & & 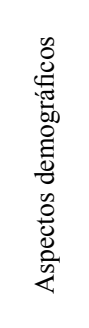 & 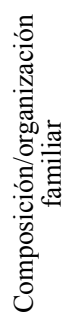 & 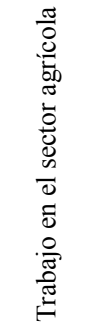 & 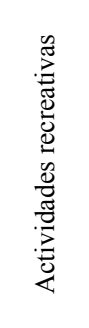 & 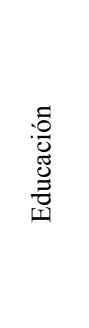 & 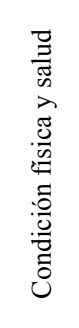 & 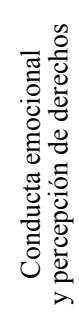 \\
\hline \multirow{2}{*}{$\begin{array}{l}\text { Aspectos } \\
\text { demográficos }\end{array}$} & $\begin{array}{l}\text { Correlación } \\
\text { de Pearson }\end{array}$ & 1 & 0.287 & 0.052 & 0.205 & 0.386 & 0.273 & -0.076 \\
\hline & Sig. (bilateral) & & 0.039 & 0.714 & 0.144 & 0.005 & 0.05 & 0.592 \\
\hline \multirow{2}{*}{$\begin{array}{l}\text { Composición / } \\
\text { organización } \\
\text { familiar }\end{array}$} & $\begin{array}{l}\text { Correlación } \\
\text { de Pearson }\end{array}$ & 0.28 & 1 & 0.395 & 0.199 & 0.358 & 0.077 & 0.217 \\
\hline & Sig. (bilateral) & 0.039 & & 0.004 & 0.157 & 0.009 & 0.587 & 0.123 \\
\hline \multirow{2}{*}{$\begin{array}{l}\text { Trabajo en el } \\
\text { sector agrícola }\end{array}$} & $\begin{array}{l}\text { Correlación } \\
\text { de Pearson }\end{array}$ & 0.052 & 0.395 & 1 & -0.146 & $0.276^{*}$ & 0.095 & 0.425 \\
\hline & Sig. (bilateral) & 0.714 & 0.004 & & 0.301 & 0.048 & 0.502 & 0.002 \\
\hline \multirow{2}{*}{$\begin{array}{l}\text { Actividades } \\
\text { recreativas }\end{array}$} & $\begin{array}{l}\text { Correlación } \\
\text { de Pearson }\end{array}$ & 0.205 & 0.199 & -0.146 & 1 & 0.213 & 0 & -0.115 \\
\hline & Sig. (bilateral) & 0.144 & 0.157 & 0.301 & & 0.13 & 1 & 0.418 \\
\hline \multirow[t]{2}{*}{ Educación } & $\begin{array}{l}\text { Correlación } \\
\text { de Pearson }\end{array}$ & 0.386 & 0.358 & $0.276^{*}$ & 0.213 & 1 & 0.183 & 0.104 \\
\hline & Sig. (bilateral) & 0.005 & 0.009 & 0.048 & 0.13 & & 0.195 & 0.465 \\
\hline \multirow{2}{*}{$\begin{array}{l}\text { Condición física } \\
\text { y salud }\end{array}$} & $\begin{array}{l}\text { Correlación } \\
\text { de Pearson }\end{array}$ & 0.273 & 0.077 & 0.095 & 0 & 0.183 & 1 & 0.153 \\
\hline & Sig. (bilateral) & 0.05 & 0.587 & 0.502 & 1 & 0.195 & & 0.28 \\
\hline \multirow{2}{*}{$\begin{array}{l}\text { Conducta emocio- } \\
\text { nal y percepción } \\
\text { de derechos }\end{array}$} & $\begin{array}{l}\text { Correlación } \\
\text { de Pearson }\end{array}$ & -0.076 & 0.217 & 0.425 & -0.115 & 0.104 & 0.153 & 1 \\
\hline & Sig. (bilateral) & 0.592 & 0.123 & 0.002 & 0.418 & 0.465 & 0.28 & \\
\hline
\end{tabular}

Fuente: elaboración propia con resultados de IBM SPSS Statistics 25.

La investigación se desarrolló conforme a los siguientes criterios: i) se obtuvo un consentimiento fundamentado de los NNA participantes en compañía de padres /tutores a los cuales se les dio a conocer la naturaleza del proceso en el que iban a participar durante la investigación, y se les informó sobre todos los resultados previstos y posibles de la investigación, ii) se estableció claramente el derecho a desistir de la participación total o parcial en alguna parte de la entrevista en cualquier momento que el menor o el padre/tutor lo desearan, iii) se definió un lenguaje y lógica en la entrevista de manera que si bien, el cuestionario elaborado contenía lenguaje técnico, este pudiera ser modificado de forma creativa y flexible por los psicólogos que lo aplicaron para abordar a los NNA en función de su edad, 
iv) Las entrevistas y cualquier otra actividad de recopilación de información que requiriera la interacción con los menores incluyendo el pesaje y medida se realizó siempre bajo la observación y visto bueno de los padres/ tutores, pero sin que estos intervinieran o influyeran en la respuesta de los NNA, v) Toda la información personal recabada en el estudio fue manejada con estricto apego a la ley federal de protección de datos personales en posesión de los particulares y cuya finalidad fue de estricto uso académico.

\section{Resultados}

\section{Aspectos demográficos}

Se observó que el género de los NNA está compuesto por 46.8 por ciento mujeres y 53.2 por ciento hombres. En términos de edad, 47 por ciento $(\mathrm{n}=224)$ de los NNA pertenecen a la categoría de 14 a 17 años, hay 334 NNA de entre 5 y 13 años (ver Tabla 3). El estudio se centró en trabajadores agrícolas que pertenecen principalmente a comunidades rurales indígenas; por lo tanto, se observó que 63 por ciento habla español, 25 por ciento zapoteco, cuatro por ciento Nahualt, dos por ciento mixteco y seis por ciento cualquier otra lengua indígena. En términos totales 37 por ciento de los NNA hablan una lengua indígena y, en algunos casos, esta es la única que conocen. Esto implica que servicios de educación y atención médica sean difíciles de brindar debido a las barreras de comunicación (Ordorica et al., 2009: 123).

Tabla 3: Sexo y edad de NNA entrevistados

\begin{tabular}{lccc}
\hline Edad & Total & Mujeres & Hombres \\
\hline 5 & 18 & 12 & 6 \\
6 & 33 & 14 & 19 \\
7 & 21 & 8 & 13 \\
8 & 43 & 19 & 24 \\
9 & 55 & 30 & 25 \\
10 & 45 & 19 & 26 \\
11 & 46 & 24 & 22 \\
12 & 32 & 15 & 17 \\
13 & 41 & 23 & 18 \\
14 & 71 & 39 & 32 \\
15 & 43 & 17 & 26 \\
16 & 52 & 19 & 33 \\
17 & 58 & 21 & 37 \\
\hline
\end{tabular}

Fuente: elaboración propia. 
Bienestar infantil y capacidades humanas entre niños, niñas y adolescentes (NNA) ... / F. PÉREZ SOTO et al.

\section{Educación}

Solo 75.6 por ciento de los NNA se encuentra actualmente inscritos en actividades académicas en los siguientes niveles: preescolar cuatro por ciento, primaria 47 por ciento, secundaria 19 por ciento y bachillerato tres por ciento. Se observó que 24.4 por ciento de los NNA $(\mathrm{n}=136)$ no estudian. Desde la perspectiva de los NNA, 93.9 por ciento consideró la educación como un factor importante, pero solo 27.8 por ciento mostró una clara comprensión de la importancia de la educación al mencionar las razones relacionadas con una mejoría a largo plazo en sus vidas. Por otro lado, 7.5 por ciento manifestó una aversión por el estudio y 2.5 por ciento consideró que la escuela como irrelevante. El 62.5 por ciento de los NNA mencionaron viajar distancias a pie superiores a los cinco kilómetros para llegar a la escuela. Las escuelas en las áreas rurales de México son de difícil acceso (Azaola-Calderón, 2010: 67). Aunque la mayoría de los NNA asisten regularmente a la escuela de lunes a viernes, 15.2 por ciento manifestó que a menudo faltan a la escuela porque hacen tareas domésticas, ayudan a sus padres en el trabajo o cuidan a sus hermanos. En términos de servicios e infraestructura educativa, hay varios aspectos críticos, en primer lugar, se observó que solo 12.2 por ciento de los NNA actualmente matriculados en la escuela, han sido apoyados con una beca lo que implica que este tipo de apoyos no llegan a ciertas comunidades rurales de México. Se observó que el solo 6.3 por ciento de NNA recibe desayuno escolar, aun cuando existe el Programa de Alimentación Escolar de México, igualmente se infiere que estos programas no llegan a las zonas rurales.

\section{Composición / organización familiar}

La familia nuclear es el tipo de familia predominante con 66.1 por ciento seguido de familia extensa con 29 por ciento, las familias mononucleares y fraternales son poco significativas con 3.4 y 1.1 por ciento, respectivamente. Cuando se cuestionó al niño sobre el establecimiento de reglas en el hogar, esta responsabilidad se dividió entre padre y madre con 44.9 y 40.7 por ciento, respectivamente. En términos de estrategias de corrección para la mala conducta, se observó que solo 27.1 por ciento de los NNA reciben un método de corrección adecuado (ver Tabla 4). El 31.5 por ciento de los NNA reciben métodos de corrección que violan sus derechos. Un modelo de educación basado en el castigo físico y las afectaciones psicológicas (insultos, humillaciones, denigración, etc.) puede conducir a condiciones mentales que pueden comprometer el desarrollo futuro y dificultar la adap- 
tación en la sociedad del niño, además de crear círculos viciosos en familias futuras. Con respecto a la calidad en la comunicación con sus padres, 79 por ciento expresó que habla constantemente con ellos, 11.6 por ciento en ocasiones y 8.2 por ciento no habla con ellos. Los principales temas abordados durante las conversaciones incluyeron 48.4 por ciento temas generales y superficiales y solo 40.3 por ciento se refiere a temas relacionados con la situación familiar o temas más profundos importantes para la educación infantil. En cuanto a la participación de los NNA en la toma de decisiones, 40.1 por ciento expresó que se le toma en cuenta a la hora de decidir las acciones dentro de la familia, aunque no se especificó el nivel y la naturaleza de su opinión. El 33.2 por ciento reportó participación nula y 23.7 por ciento en ocasiones.

Tabla 4: Acciones correctivas para mala conducta de NNA

\begin{tabular}{lcc}
\hline Acción & Frecuencia & Porcentaje \\
\hline El niño es golpeado, insultado, gritado, ignorado & 176 & 31.5 \\
El niño es regañado sin más castigo & 221 & 39.6 \\
Los padres hablan con el niño y se establece & 151 & 27.1 \\
un castigo razonable & 10 & 1.8 \\
Sin respuesta &
\end{tabular}

Fuente: elaboración propia.

\section{Actividades recreativas}

En la frecuencia de juego se observó 74.7 por ciento $(n=413)$ con actividad de una o dos veces al día, 19.7 por ciento $(\mathrm{n}=109)$ juega más de tres veces al día, sin embargo, 5.2 por ciento $(\mathrm{n}=29)$ expresó no tener permitido el juego. En términos de la participación de los padres en actividades recreativas con hijos, se observó que solo 44.4 por ciento tienen este tipo de interacción con ellos. De la muestra que respondió afirmativamente, solo 26.7 por ciento mencionó que toda la familia participa en las actividades recreativas y juegos. El 33.5 por ciento interactúa solo con uno de los padres. En términos del tipo de juegos y actividades, se observó que 93.9 por ciento practica al menos una actividad lúdica, esto refleja que, aunque la participación de los padres en actividades recreativas es baja, tales actividades incluyen juegos que favorece la autoconfianza, la autonomía y la formación de la personalidad. Esto se evidenció también cuando los NNA fueron interrogados sobre el sentimiento / sensación / percepción que las 
actividades recreativas causan en ellos, donde 78.5 por ciento expresaron sentimientos y pensamientos positivos que denotan alegría.

\section{Trabajo en el sector agrícola}

En la primera instancia, se identificó que, de la muestra de 558 NNA, solo 74.3 por ciento $(n=415)$ acompañan a sus padres al campo (parcelas agrícolas). Las actividades que realizan los NNA en el campo de trabajo son diversas y van desde solo el acompañamiento hasta el trabajo físico (ver Tabla 5).

Tabla 5: Actividades desempeñadas por los NNA en las parcelas agrícolas

\begin{tabular}{lcc}
\hline Actividad & Frecuencia & Porcentaje \\
\hline A1. Solo acompaña a los padres/familiares & 10 & 2.4 \\
A2. Brinda ayuda (labores generales) & 175 & 42.2 \\
A3. Trabaja (Labores agrícolas) & 204 & 49.1 \\
A4. Cuida a hermanos/familiares & 11 & 2.7 \\
A5. Hace actividades recreativas & 15 & 3.6 \\
\hline
\end{tabular}

Fuente: elaboración propia.

La Tabla 5 muestra un alto porcentaje de NNA que realizan actividades inesperadas para su edad. Se observa que, en términos generales, 91.3 por ciento realiza actividades laborales, particularmente, 50.5 por ciento realiza actividades de cosecha que implican un trabajo físico arduo. Por su parte la Tabla 6 muestra los datos desagregados en edad y sexo de las cinco actividades que realizan los NNA en las parcelas agrícolas. Es posible apreciar que en la actividad A2 y A3 que implican trabajo diverso concentra mayormente a hombres con una razón cercana a 1.5 hombres por cada mujer. Igualmente se observó que en las diferentes actividades se concentra mayormente los NNA entre 13 a 17 años.

Por otra parte, la frecuencia de las actividades laborales en los NNA evidenció una presencia constante en el campo de trabajo, esto es no solo en fines de semana sino también de lunes a viernes. El sábado concentra la mayoría de los casos y el domingo la minoría. Teniendo en cuenta que las actividades laborales incluyen trabajo físico, se observó que 57 por ciento trabajan durante cuatro o más horas al día. En términos de retribución económica, solo 371 NNA reciben un pago por su trabajo (58.5 por ciento de un total de 558) ya sea de forma periódica o esporádica. El monto del pago en su mayoría no supera los cinco dólares americanos (ver Tabla 7). 
Tabla 6: Datos desagregados en edad y sexo de actividades en parcelas agrícolas (Número de NNA)

\begin{tabular}{crrrrrrrrrrr}
\hline \multirow{2}{*}{ Edad } & \multicolumn{1}{c}{$\mathrm{A} 1$} & \multicolumn{1}{c}{$\mathrm{A} 2$} & \multicolumn{1}{c}{$\mathrm{A} 3$} & \multicolumn{2}{c}{$\mathrm{A} 4$} & $\mathrm{~A} 5$ & \\
& $\mathrm{M}$ & $\mathrm{H}$ & $\mathrm{M}$ & $\mathrm{H}$ & $\mathrm{M}$ & $\mathrm{H}$ & $\mathrm{M}$ & $\mathrm{H}$ & $\mathrm{M}$ & $\mathrm{H}$ & Total \\
\hline 5 & 1 & 1 & 3 & 1 & 2 & 3 & 0 & 0 & 1 & 0 & 12 \\
6 & 0 & 0 & 4 & 16 & 1 & 1 & 1 & 0 & 1 & 4 & 28 \\
7 & 0 & 0 & 1 & 3 & 0 & 1 & 0 & 1 & 0 & 0 & 6 \\
8 & 1 & 1 & 5 & 8 & 1 & 4 & 0 & 2 & 1 & 1 & 24 \\
9 & 1 & 0 & 15 & 10 & 1 & 2 & 1 & 1 & 0 & 2 & 33 \\
10 & 0 & 1 & 6 & 11 & 4 & 5 & 0 & 2 & 0 & 0 & 29 \\
11 & 0 & 0 & 10 & 8 & 2 & 6 & 1 & 0 & 0 & 2 & 29 \\
12 & 0 & 1 & 4 & 10 & 5 & 2 & 0 & 0 & 0 & 1 & 23 \\
13 & 0 & 0 & 7 & 9 & 8 & 6 & 0 & 0 & 1 & 0 & 31 \\
14 & 0 & 0 & 14 & 4 & 11 & 26 & 0 & 0 & 0 & 0 & 55 \\
15 & 1 & 1 & 5 & 5 & 8 & 19 & 1 & 0 & 0 & 0 & 40 \\
16 & 0 & 0 & 7 & 2 & 10 & 30 & 0 & 0 & 0 & 0 & 49 \\
17 & 1 & 0 & 1 & 6 & 16 & 30 & 1 & 0 & 0 & 1 & 56 \\
Total & 5 & 5 & 82 & 93 & 69 & 135 & 5 & 6 & 4 & 11 & 415 \\
\hline
\end{tabular}

Fuente: elaboración propia.

Tabla 7: Retribución económica por el trabajo

\begin{tabular}{lrr}
\hline Cantidad & Frecuencia & Porcentaje \\
\hline Menos de 5 dólares americanos diarios & 210 & 56.5 \\
Más de 5 dólares americanos diarios & 123 & 33.3 \\
No lo sabe & 6 & 1.6 \\
No contestó & 32 & 8.6 \\
Total & 371 & 100.0 \\
\hline
\end{tabular}

Fuente: elaboración propia.

Por otra parte, la frecuencia de las actividades laborales en los NNA evidenció una presencia constante en el campo de trabajo, esto es no solo en fines de semana sino también de lunes a viernes. El sábado concentra la mayoría de los casos y el domingo la minoría. Teniendo en cuenta que las actividades laborales incluyen trabajo físico, se observó que 57 por ciento trabajan durante cuatro o más horas al día. En términos de retribución eco- 
nómica, solo 371 NNA reciben un pago por su trabajo (58.5 por ciento de un total de 558) ya sea de forma periódica o esporádica. El monto del pago en su mayoría no supera los cinco dólares americanos (ver Tabla 7).

De la retribución económica mostrada en la Tabla 7, se observó que de 371 NNA que ayudan y/o trabajan en las parcelas agrícolas, solo 36.8 por ciento reciben el pago ellos mismos, 35.9 por ciento indica que el pago es recibido por sus padres, y el porcentaje restante indicó que el dinero lo recibe otro familiar o desconoce el destino final del pago. Del porcentaje de NNA que recibe un pago efectivo, este ingreso en gastado principalmente en vestimenta (ver Figura 1).

Figura 1: Destino del dinero ganado por los NNA

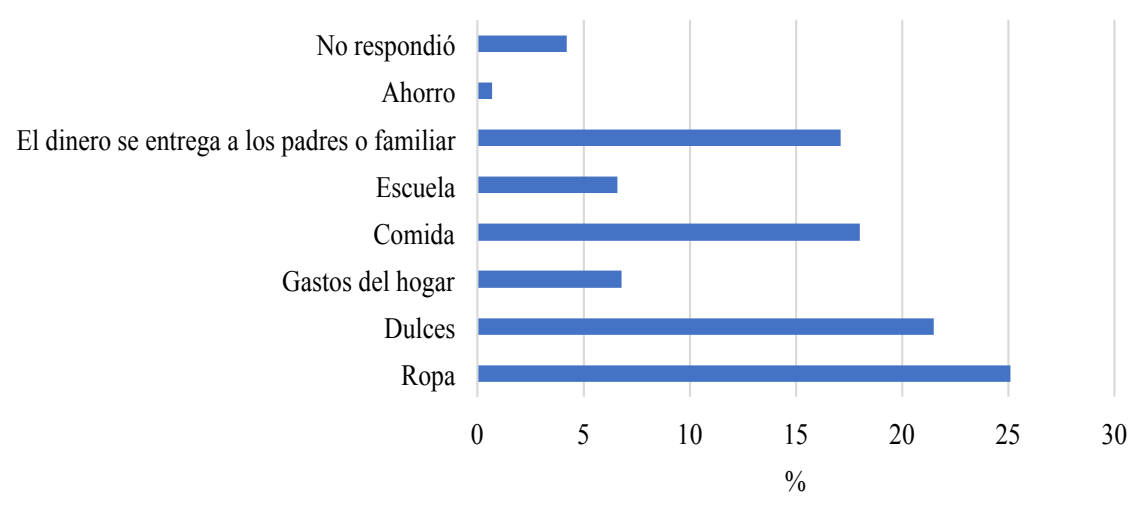

Fuente: elaboración propia.

Con respecto a la vida laboral, se observó que los NNA tienden a comenzar actividades laborales desde muy temprana edad. El 10.5 por ciento comienza a trabajar a la edad de cinco años, mientras que más de 60 por ciento inicia a laborar entre los seis y diez años. Cabe señalar que la participación de menores en actividades agrícolas está prohibida dado el esfuerzo físico necesario para llevar a cabo las tareas. Además, el tipo de equipo y maquinaria utilizados en dichas actividades crea un entorno peligroso. Se identificó que los NNA que trabajan en tierras de cultivo manejan equipos especiales que aumentan los riesgos, estos elementos incluyen: machetes, palas, tractores, pulverizadores, pesticidas, picos, tijeras, hachas, cuchillos, rastrillos de jardín, y azadones. El trabajo no siempre es supervisado por adultos, lo que aumenta el riesgo de accidentes. En cuanto a la ocurrencia de accidentes, se observó que las caídas y heridas por objetos punzocortantes son los accidente con mayor ocurrencia (ver Figura 2). 
Figura 2: Principales accidentes ocurridos en las jornadas de trabajo

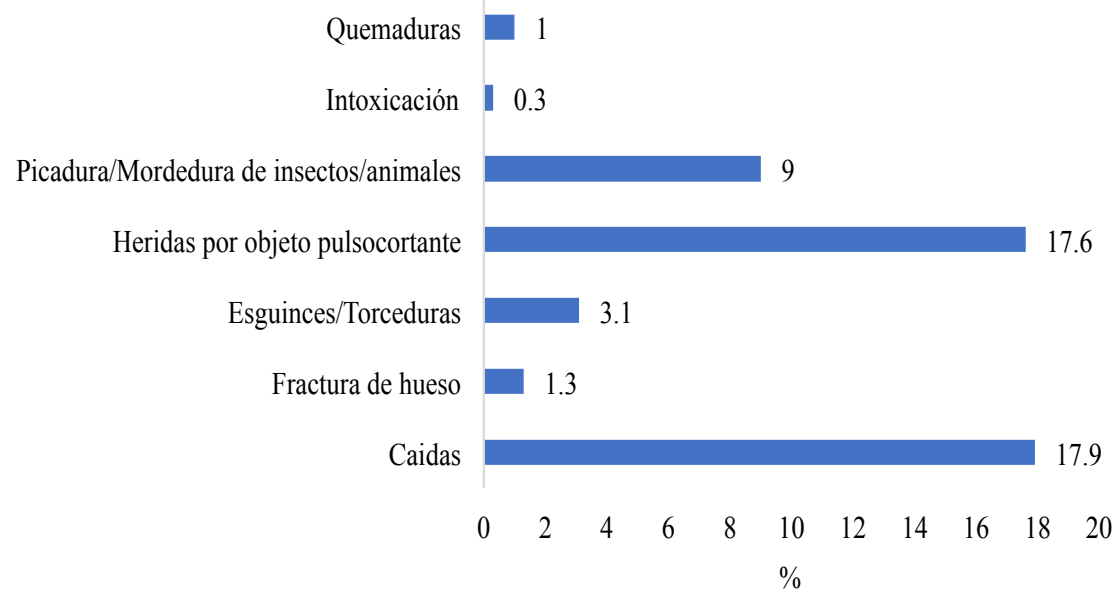

Fuente: elaboración propia.

El tipo de accidentes que han sufrido los NNA está en función del rango de edad y el sexo, lo cual guarda relación con la mayor concentración de edades de 14-17 años en las actividades físicas demandantes (ver Tabla 6). En la Figura 3 es posible observar el porcentaje de ocurrencia de los accidentes descritos en la Figura 2 pero de forma desagregada por edad y sexo. Resalta el hecho que el rango de edad de 14-17 presenta la mayor cantidad de ocurrencia de accidentes con mayor grado de seriedad como fracturas, torceduras, intoxicaciones y quemaduras. Por otra parte, se observa que las caídas, cortadas y las picaduras/mordeduras de insectos presentan una mayor heterogeneidad en la distribución, siendo este tipo de accidentes los que más se presentan en todos los rangos de edad y sexo.

Adicional a la ocurrencia de accidentes, se observó un conjunto de diferentes consecuencias para el trabajo, esto incluyó dolor muscular (19.5 por ciento), fatiga (50.6 por ciento), insolación (8.6 por ciento) y mareos (5.3 por ciento), como se puede observar en la Figura 3. Finalmente, los NNA mostraron una variedad de percepciones con respecto a la actividad laboral llevada a cabo. Al ser cuestionados sobre la razón para laborar con sus padres, 46.9 por ciento expresó un deseo de trabajar para imitar a sus familiares, mientras que 25.1 por ciento evidenció la necesidad de trabajar dadas las condiciones económicas de su familia. El 8.2 por ciento expresó que son obligados a trabajar sin preguntar opiniones. 
Bienestar infantil y capacidades humanas entre niños, niñas y adolescentes (NNA) ... / F. PÉREZ SOTO et al.

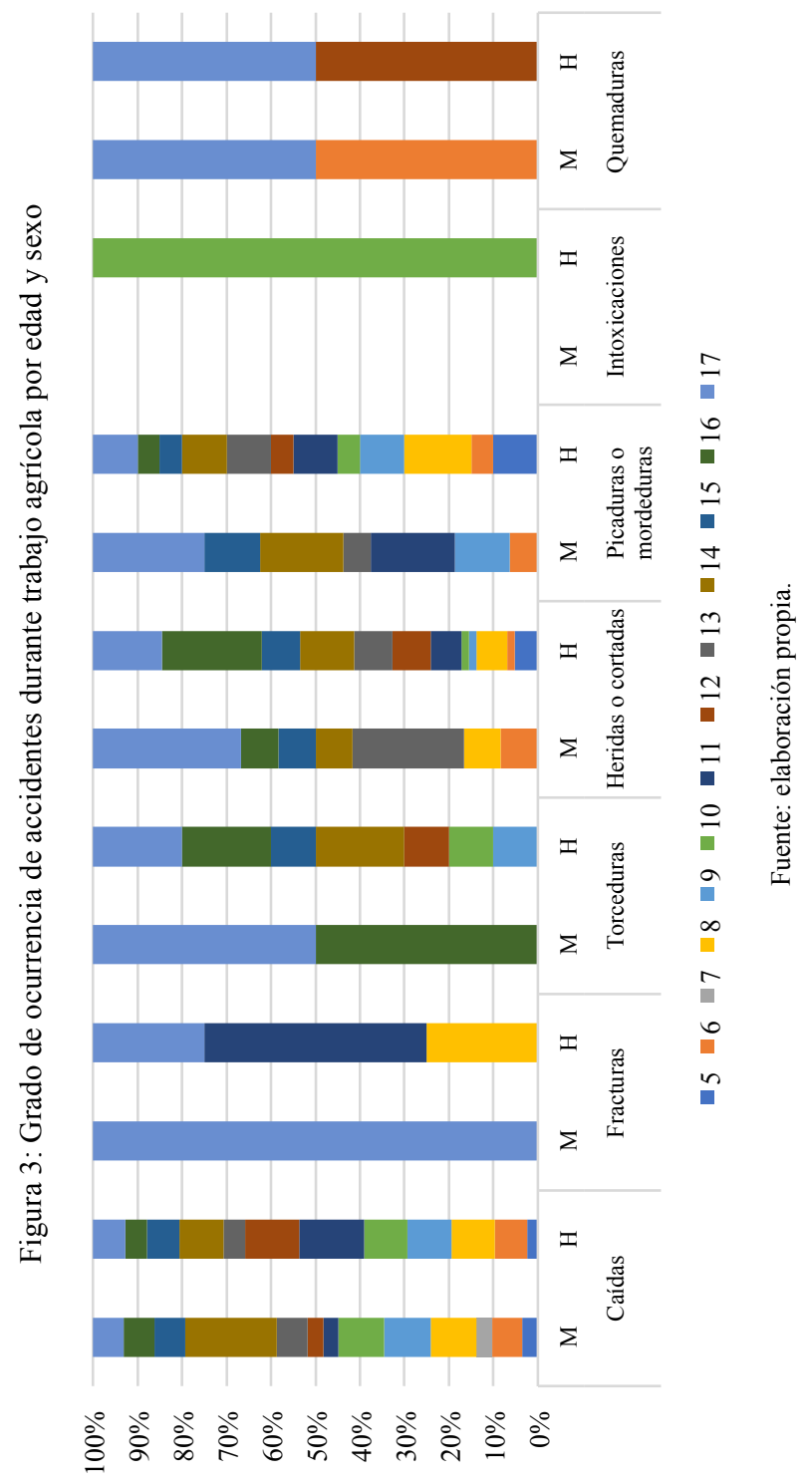




\section{Condición física y cuidado de la salud}

Los aspectos relacionados con las condiciones físicas y la atención médica incluyeron principalmente valores de peso y altura de los NNA con el fin de identificar desviaciones en los estándares esperados para cada edad. Las mediciones relacionadas con el peso y la altura resultaron de la siguiente manera (Tabla 8):

Tabla 8: Estadísticas de peso y estatura en NNA

\begin{tabular}{lcccc}
\hline Condición & \multicolumn{2}{c}{ Peso } & \multicolumn{2}{c}{ Estatura } \\
\hline & Frecuencia & Porcentaje & Frecuencia & Porcentaje \\
\hline $\begin{array}{l}\text { Por debajo del peso esperado } \\
\text { para la edad o con sobrepeso }\end{array}$ & 190 & 34.1 & 108 & 19.4 \\
$\begin{array}{l}\text { Dentro de la media normal } \\
\text { para la edad }\end{array}$ & 358 & 64.2 & 443 & 79.4 \\
No analizados & 10 & 1.8 & 7 & 1.3 \\
\hline
\end{tabular}

Fuente: elaboración propia.

Se puede observar que una cantidad importante de NNA (34.1 en peso y 19.4 por ciento en altura) mostró una desviación en los niveles estándares, esto implica una dieta inadecuada y desequilibrada que puede ser el resultado de recursos económicos insuficientes para acceder a alimentos saludables. De forma particular en la Tabla 9 se puede apreciar la participación porcentual en cada categoría de desviaciones en peso y estatura tanto por edad y sexo.

Se observó que, en términos de nutrición, solo 19.7 por ciento tiene una dieta balanceada en ausencia de alimentos con una alta concentración de grasa y azúcar. En cuanto a las enfermedades, entre los principales padecimientos se identificaron a las enfermedades respiratorias con 42.2 por ciento, enfermedades gastrointestinales con 10.3 por ciento, molestias en ojos/oídos/dientes con 5.9 por ciento y la irritación de la piel (sarpullido) con 1.5 por ciento. El 2.3 por ciento presentaron infestación de piojos. Se observó que solo 44.6 por ciento de los NNA recibe atención médica. En términos de calidad del servicio médico, 84.5 por ciento percibe que la asistencia es aceptable, aunque no siempre se brindan los medicamentos. 
Bienestar infantil y capacidades humanas entre niños, niñas y adolescentes (NNA) ... / F. PÉREZ SOTO et al.

Tabla 9: Participación porcentual en peso y estatura por rango de edades y sexo

\begin{tabular}{lrrrrrrrr}
\hline & \multicolumn{4}{c}{ Peso } & \multicolumn{4}{c}{ Estatura } \\
& \multicolumn{1}{c}{$\begin{array}{c}\text { Fuera de la } \\
\text { media }\end{array}$} & \multicolumn{2}{c}{$\begin{array}{c}\text { Dentro de la } \\
\text { media }\end{array}$} & \multicolumn{2}{c}{ Fuera de la } & \multicolumn{2}{c}{$\begin{array}{c}\text { Dentro de la } \\
\text { media }\end{array}$} \\
Edad & M & H & M & H & M & H & M & H \\
\hline 5 & 27.8 & 22.2 & 38.9 & 11.1 & 16.7 & 11.1 & 50.0 & 22.2 \\
6 & 16.1 & 16.1 & 29.0 & 38.7 & 0.0 & 12.9 & 41.9 & 45.2 \\
7 & 23.8 & 28.6 & 61.9 & 81.0 & 9.5 & 28.6 & 81.0 & 85.7 \\
8 & 12.2 & 14.6 & 31.7 & 41.5 & 4.7 & 14.0 & 39.5 & 41.9 \\
9 & 11.1 & 9.3 & 44.4 & 35.2 & 9.4 & 3.8 & 45.3 & 41.5 \\
10 & 11.1 & 6.7 & 33.3 & 48.9 & 2.2 & 6.7 & 42.2 & 48.9 \\
11 & 17.4 & 21.7 & 34.8 & 26.1 & 4.3 & 6.5 & 47.8 & 41.3 \\
12 & 12.5 & 28.1 & 34.4 & 25.0 & 0.0 & 12.5 & 46.9 & 40.6 \\
13 & 22.5 & 27.5 & 35.0 & 15.0 & 15.0 & 20.0 & 42.5 & 22.5 \\
14 & 15.5 & 16.9 & 39.4 & 28.2 & 8.5 & 9.9 & 46.5 & 35.2 \\
15 & 14.6 & 29.3 & 22.0 & 34.1 & 9.3 & 14.0 & 30.2 & 46.5 \\
16 & 12.0 & 24.0 & 26.0 & 38.0 & 8.0 & 14.0 & 30.0 & 48.0 \\
17 & 17.2 & 24.1 & 19.0 & 39.7 & 19.0 & 15.5 & 17.2 & 48.3 \\
\hline
\end{tabular}

Fuente: elaboración propia.

\section{Comportamiento y percepción de los derechos}

Las opiniones proporcionadas por los NNA se categorizaron en elementos que pueden mostrar el estado actual del conocimiento y la comprensión de los derechos básicos de los NNA.

La Tabla 10 muestra la percepción con respecto a la expectativa que los NNA tienen de sus propios derechos. Se observó que, en términos generales, 80.4 por ciento ( 449 de 558) dieron una respuesta que reflejaba una situación real esperada para su edad en la que no se violaban sus derechos. Esta respuesta agrupaba opciones como derecho a comer, a jugar, estudiar, tener ropa entre otras declaraciones que sean consistentes con lo establecido en la Declaración de los Derechos del Niño. 
Tabla 10. Afirmación: Tengo derecho a...

\begin{tabular}{lrr}
\hline Percepción & Frecuencia & Porcentaje \\
\hline Ningún aspecto que vulnere sus derechos & 63 & 11.3 \\
Uno o dos aspectos dentro de sus derechos & 258 & 46.2 \\
Más de dos aspectos dentro de sus derechos & 191 & 34.2 \\
No sabe & 25 & 4.5 \\
No respondió & 21 & 3.8 \\
\hline
\end{tabular}

Fuente: elaboración propia.

Por otro lado, 44.1 por ciento $(n=246)$ consideraron que los NNA pueden trabajar siendo menores de 14 años. Sin embargo, los datos de la Tabla 11 muestran que los NNA perciben el trabajo como algo natural / normal para su edad, esto debido al entorno en el que crecen y a los modelos de rol que observan entre hermanos y amigos.

Tabla 11: Afirmación: Un niño puede trabajar a la edad de...

\begin{tabular}{lrr}
\hline Edad & Frecuencia & Porcentaje \\
\hline Una edad menor de los 14 años & 246 & 44.1 \\
A los 14 años & 202 & 36.2 \\
Un niño no debe trabajar & 64 & 12.0 \\
No sabe & 26 & 4.7 \\
No respondió & 17 & 3.0 \\
\hline
\end{tabular}

Fuente: elaboración propia.

La Tabla 12 muestra que, aunque los NNA perciben el trabajo como una actividad normal para ellos, en relación con su preferencia, la idea principal que apareció fue el estudio. El 40.7 por ciento manifestó que preferiría ir a la escuela. Esto es congruente con los resultados presentados en la sección 3.2 en los que los NNA consideraron la escuela como un factor importante. El comportamiento de los NNA también fue analizado. Se analizó la reacción de los NNA y cómo interactúan con las mascotas para centrarse en los sentimientos, emociones y actitudes mostradas.

La Tabla 13 enumera las categorías de conducta medida, se puede observar que, a pesar del contexto y las condiciones de los NNA, los sentimientos de amor y alegría son más elevados que los sentimientos de ira, miedo y tristeza. Sin embargo, 83 NNA mostraron una conducta negativa hacia los animales con una presencia significativa de sentimientos de enojo. 
Tabla 12: Afirmación: Yo preferiría...

\begin{tabular}{lrr}
\hline Preferencia & Frecuencia & Porcentaje \\
\hline Ir a la escuela & 227 & 40.7 \\
Estar en casa & 32 & 5.8 \\
Ir al campo agrícola & 102 & 18.3 \\
Jugar/divertirme & 108 & 19.3 \\
Regresar a mi lugar de origen & 42 & 7.6 \\
Otro & 40 & 7.2 \\
No respondió & 7 & 1.2 \\
\hline
\end{tabular}

Fuente: elaboración propia.

Tabla 13: Emociones and actitudes mostradas por los NNA

\begin{tabular}{lcc}
\hline Categoría & Frecuencia & Porcentaje \\
\hline $\begin{array}{l}\text { Los sentimientos de amor y felicidad se pre- } \\
\text { sentan en mayor proporción que los sentimien- }\end{array}$ & 289 & 51.8 \\
$\begin{array}{l}\text { tos de tristeza, miedo y enojo. } \\
\begin{array}{l}\text { Todos los sentimientos están en proporción } \\
\text { promedio }\end{array}\end{array}$ & 181 & 32.4 \\
$\begin{array}{l}\text { Los sentimientos de tristeza, miedo y enojo se } \\
\text { presentan en mayor proporción que los senti- } \\
\text { mientos de amor y felicidad }\end{array}$ & 83 & 14.9 \\
No respondió & 5 & 0.9 \\
\hline
\end{tabular}

Fuente: elaboración propia.

\section{DiscuSIÓN}

La encuesta realizada como se explica en la sección de metodología se categorizó en siete elementos para las respuestas grupales. Estos elementos se basaron en las capacidades humanas centrales de Nussbaum, por lo tanto, cada capacidad se contrasta con los resultados obtenidos de la siguiente manera:

1. Vida. Esta capacidad argumenta que una persona, en este caso un niño, debe poder vivir en condiciones normales de su contexto. Los resultados mostraron que, aunque no se han producido víctimas mortales y que los NNA no corren el riesgo de morir prematuramente, el tipo de actividades y condiciones a las que están expuestos por el trabajo comprometen su seguridad a la vida. Si es importante hacer la aclaración 
que en los accidentes de trabajo se observó una mayor concentración de edades de entre 14 y 17 años por lo que los niños de menor rango de edad no están expuestos de forma importante a riesgos que comprometan su seguridad a la vida. Sin embargo, es imperativo minimizar cualquier riesgo que atente contra esta capacidad humana.

2. Salud corporal. Los NNA deben gozar de buena salud; estar adecuadamente nutrido; tener un refugio adecuado. Esta capacidad no es desarrollada por cierto porcentaje de los NNA que participaron en el estudio. Los resultados mostraron que la salud de varios NNA no es óptima. Se observaron medidas de peso y estatura inferiores a las esperadas para su edad. Esto implica una alimentación precaria derivado tanto las carencias económicas de las familias, así como del limitado acceso a los programas gubernamentales de becas y desayunos escolares. Aunque las enfermedades graves no han afectado a los NNA, se observaron puntos críticos como presencia de ftirápteros (piojos). Cabe señalar que los piojos se propagan fácilmente, especialmente entre los NNA en edad escolar. Esta infestación puede significar falta de limpieza o condiciones insalubres (Goodman et al., 2013: 2398) y puede provocar frustración psicológica para padres e hijos; además, las prácticas preventivas y terapéuticas, como el afeitado de la cabeza y la exclusión de actividades escolares pueden inducir estrés social (Falagas et al., 2008: 1493). En consecuencia, esta capacidad no es lograda satisfactoriamente y repercute en el bienestar y desarrollo de los infantes. Para su mejoramiento es fundamental implementar estrategias en los diversos niveles de gobierno para asegurar que tanto los programas gubernamentales de asistencia social, así como el acceso al sistema de salud pública sean garantizados a estas poblaciones vulnerables.

3. Integridad corporal. Esta capacidad destaca que las personas deben poder moverse libremente de un lugar a otro; bajo condiciones soberanas y de decisión propia. Este aspecto se aplica a los NNA, pero de una manera completamente diferente. Los NNA, como hijos de trabajadores agrícolas migrantes, deben mudarse de un lugar a otro dadas las condiciones de trabajo de sus padres. Por lo tanto, la movilidad de las familias de migrantes agrícolas (incluidos los NNA) se da por una necesidad económica de subsistencia, no se genera por libre voluntad.

4. Sentidos, imaginación y pensamiento. Esta capacidad implica el uso de sentidos, poder imaginar, pensar y razonar adecuadamente. Esto involucra recibir una educación adecuada, que incluye, entre otros, la alfabetización y la formación matemática y científica básica. También 
involucra la elección propia sobre religión, gustos literarios, de música, etc. Esta capacidad está escasamente presente en los NNA. Los resultados evidenciaron que, aunque todos los NNA encuestados están actualmente matriculados en cursos académicos, la combinación con las actividades laborales puede llevar a un creciente ausentismo y deserción escolar alineándose así con lo expuesto por Cavalcanti (2003: 741). De forma adicional, es necesario precisar que las condiciones adversas para el acceso a escuelas en zonas rurales tanto en el lugar de origen de los NNA como en los lugares donde migran con sus familias a trabajar, es una situación recurrente en México. Como lo exponen Juárez y Rodríguez (2016:1) existen diversos factores que afectan a la equidad educativa en escuelas rurales de México, desde la infraestructura precaria hasta problemas de discriminación racial. Por tanto, el panorama adverso para el acceso a la educación de los NNA analizados no es una situación asilada, sino que predomina en gran parte del sector rural del país. Además, la capacidad de creatividad en los NNA es en su mayoría reprimida por el sistema educativo actual no solo entre NNA trabajadores de zonas rurales sino los NNA en general de México. Cuando un niño comienza su educación en los niveles preescolar y primaria, los pensamientos creativos son altamente valorados y se incentiva al niño a cuestionar, tocar y experimentar con su entorno para que su enseñanza sea lúdica y didáctica. Sin embargo, cuando este mismo infante llega a la educación secundaria y media superior, las preguntas creativas comienzan a descender y el pensamiento creativo es reemplazado por un pensamiento analítico, que siempre busca el análisis y la resolución de problemas, pero basado en cuestiones realistas que siempre se apegan al estándar de las cosas (Martínez-Villegas et al., 2017: 87). Esta capacidad central es fundamental debido al impacto que puede tener en el futuro desarrollo personal de los NNA. El bienestar y la calidad de vida una vez que los NNA se han convertido en adultos están vinculados a su capacidad cognitiva desarrollada durante la infancia. La capacidad creativa no es un elemento innato solo de personas talentosas en música, artes o ciencias. En consecuencia, no hay personas creativas y personas no creativas, simplemente hay personas que han logrado desarrollar esa parte de la mente (Rogers, 1954: 249). Para que los NNA logren un desarrollo personal adecuado y un bienestar futuro, deben formarse de acuerdo con sus capacidades particulares (inteligencia). No obstante, los modelos de educación actuales ignoran el hecho de que los NNA pueden poseer diferentes tipos 
de inteligencias. La teoría de las inteligencias múltiples sugiere que hay ocho inteligencias diferentes: musical-rítmica; visual-espacial; lingüística verbal; lógica matemática; corporal-cinestésico; interpersonal; intrapersonal y naturalista (Gardner, 1989: 1989). El argumento de Gardner ha sido debatido en múltiples investigaciones (Gilman, 2012: 18; Geake, 2008: 123). Sin embargo, existe un grado de aceptación en la comunidad científica y académica sobre la tipología propuesta. Es necesario enfatizar que los NNA desarrollan su tipo de inteligencia de una manera y en un nivel particular, producto de la dotación biológica del individuo, de su interacción con el entorno y la cultura prevaleciente en su momento histórico. Sin embargo, el desarrollo de esta inteligencia, que está relacionada en gran medida con el bienestar, es una función del grado de estimulación y del entorno en el que se desarrolla el niño. Por lo tanto, las condiciones actuales de los NNA trabajadores en zonas rurales son desalentadoras y difícilmente se logrará el bienestar y el desarrollo personal en el futuro. Los resultados evidenciaron que la provisión de alternativas recreativas, deportivas y/o culturales es inexistente. Igualmente, los padres, de forma muy limitada, propician actividades lúdicas para sus hijos. Si bien dadas las condiciones económicas de las familias, es entendible la imposibilidad de brindar alternativas para el fomento deportivo y cultural del niño, la convivencia lúdica de padres e hijos puede ser realizada sin necesidad de recursos económicos. Tales elementos pueden ayudar a los NNA a desarrollar sus mentes y así lograr esta capacidad central.

5. Emociones. Esta capacidad se refiere a poder desarrollar apegos emocionales hacia personas o bienes materiales; implica la capacidad de amar, llorar, experimentar anhelo, gratitud o bien ira y enojo justificados. Los NNA mostraron un equilibrio adecuado con respecto a esta capacidad. Solo 14.9 por ciento $(\mathrm{n}=83)$ manifestaron mayores sentimientos de ira, tristeza y miedo sobre las emociones de positivas. El estudio no se enfocó en el origen psicológico de tales emociones, sin embargo, el ambiente, las limitaciones económicas y la composición familiar pueden afectar el estado emocional de los NNA. Además, la interacción de los padres con las emociones mostradas por los NNA puede tener un impacto en su inteligencia emocional futura. Si los padres reaccionan de manera negativa ante las emociones expresadas por los NNA puede impactar significativamente su desarrollo. Es importante que un niño pueda desarrollar un nivel de confianza para expresar sus emociones y esto está en función del trato que reciben de las perso- 
nas en su entorno, particularmente padres o tutores (Hamada-Basset et al., 2016: 4). Esto implica que los padres deben prestar atención a las emociones expresadas por sus hijos, de lo contrario, una sobrecarga/ extraversión de alto temperamento puede llevar a los NNA a exhibir hiperactividad y agresión (Berdan et al., 2009: 957), comprometiendo el logro de esta capacidad central.

6. Razón práctica. Esta capacidad implica formar una concepción del bien y participar en una reflexión crítica sobre la planificación de la vida propia. En el estudio, esta capacidad se vinculó a la participación de los NNA en la toma de decisiones dentro de la familia. Se observó que solo 40 por ciento de los NNA son tomados en consideración, aunque no se especificó el nivel y la naturaleza de la participación. El bajo porcentaje de NNA que es incluido y escuchado por sus padres manifiesta un precario desarrollo de esta capacidad central. Su fomento debe incrementarse ya que, si los NNA perciben que sus ideas son escuchadas, además de la inclusión de los NNA, la valorización de sus opiniones puede alentar un pensamiento crítico y participativo en su futuro.

7. Afiliación. Esta capacidad implica poder vivir con otras personas, reconocer y mostrar interés por otros seres humanos, participar en diversas formas de interacción social. Esta capacidad se midió al analizar la interacción de los NNA con los padres. Los resultados mostraron que menos de la mitad de los NNA interactúan con una contraparte, ya sea padres o familiares cercanos, esto también está relacionado con la capacidad de razón práctica dado que pocos NNA se incorporan en las decisiones familiares. Esta situación puede disminuir el grado de afiliación de los NNA. Además, el simple hecho de que los NNA se ven obligados a trabajar en actividades agrícolas implica que los NNA no son tratados como seres con capacidades de razonamiento y elección, lo que compromete el desarrollo personal.

8. Otras especies. Se refiere a la posibilidad de vivir considerando el bienestar de otras especies, como animales, plantas y los ecosistemas en general. Esta dimensión se analizó al medir el tipo de sentimiento expresado cuando se interactúa con mascotas. Se observó que los NNA tienden a presentar una interacción positiva con los animales y la naturaleza, dado el entorno natural donde las familias agrícolas interactúan lo que potencia su desarrollo. Estos resultados son consistentes con lo reportado por Power (2000: 55) quien menciona que la constante interacción con animales y naturaleza impacta positivamente la persona- 
lidad de menores al desarrollar una conciencia sobre la riqueza de los recursos naturales y el valor a la vida. En consecuencia, esta capacidad se desarrolla de forma efectiva en los menores.

9. Recreación. Esta capacidad implica el poder reír, jugar y disfrutar de actividades recreativas. Esta capacidad se vincula con la capacidad de sentidos, imaginación y pensamiento dado que la recreación y los juegos tiene como objetivo fomentar las emociones y pensamientos positivos. Se observó que la mayoría de los NNA juegan y disfrutan actividades recreativas al menos una vez al día, aunque la participación de padres y/o familiares es limitada. Fomentar actividades recreativas físicas, artísticas y al aire libre, especialmente en grupos, puede llevar a los NNA a lograr una mejora significativa en el valor general de la resiliencia (Romero-Barquero y Saavedra-Guajardo, 2016: 43). En consecuencia, aunque actualmente esta capacidad se desarrolla ya que los menores en su mayoría realizan actividades recreativas, si es importante señalar que para su mejoramiento se debe sensibilizar a los padres/tutores de la importancia que tiene el acompañamiento de este tipo de actividades.

10. Control sobre el medio ambiente. Esto capacidad central se divide en dos dimensiones: la dimensión política y la dimensión material. La dimensión política implica poder participar de manera efectiva en las elecciones políticas que gobiernan la vida de uno, mientras que la dimensión material implica poder mantener la propiedad de los bienes. Con respecto a estos aspectos, los NNA, aunque no son parte de la vida política del país, sí son miembros integrantes de una familia dentro de la cual se toman decisiones, sin embargo, quedó demostrado que gran parte de los NNA no tienen injerencia en decisiones dentro del hogar y su opinión no es tomada en cuenta (ver capacidad de razón práctica). Para incentivar el desarrollo de esta capacidad es necesaria la sensibilización hacía los jefes de familia, ya que como lo expone Torres et al. (2008:31) la integración de todos los miembros de la familia en las decisiones no solo puede potenciar aptitudes como la seguridad, la autoestima y la madurez, sino que favorece la equidad de género al valorar en partes iguales las opiniones de hombres y mujeres en la familia, lo que genera un modelo adecuado de inclusión a los menores. Por otra parte, en lo que se refiere al control material, los NNA no poseen propiedades, sin embargo, si se evidenció que, en un elevado número de casos, los ingresos por el trabajo que desempeñan no llegan a ellos y son cobrados por una tercera parte. Este aspecto, si bien se puede inter- 
pretar como una vulneración a la posibilidad de control sobre el fruto económico de un trabajo, debe ser analizado de manera más profunda en términos de la aplicación final del dinero. Los resultados mostraron que, si bien la gran mayoría de los NNA no administran ellos mismo los salarios obtenidos, si fue evidente el hecho que los padres/tutores destinan dicho recurso al sustento familiar, o bien es utilizado en la compra de ropa y vestido y otras necesidades escolares de los menores. Esto es consistente con lo mencionado por Salazar (1996:1) quien argumenta que sobre en todos en menores trabajadores entre 13 y 17 años, su contribución a la economía familiar es significativa y aunque el trabajo debería estar ausente en esta población, su existencia ayuda a la subsistencia de todos los miembros en el núcleo familiar. Por tanto, esta capacidad es desarrollada parcialmente.

\section{Conclusiones}

El caso de los NNA analizados, hijos de trabajadores agrícolas migrantes en México, presenta aspectos críticos que comprometen el desarrollo de las capacidades centrales humanas propuestas por Nussbaum. La investigación realizada mostró que, si bien en su mayoría, los NNA se ven en la necesidad de realizar actividades laborales que a menudo implican un trabajo físico arduo que pone en riesgo su integridad física, sí se identificó una diferencia marcada entre el trabajo infantil y el trabajo infantil en condiciones de explotación. En el caso analizado se observó que las condiciones de trabajo infantil vulneran los derechos de menores, pero que estas acciones no están relacionadas con una explotación de la persona, sino que obedecen a las condiciones estructurales tanto económicas como sociales en las que se desarrolla el fenómeno de la migración agrícola en zonas rurales de múltiples familias mexicanas. Bajo este contexto los NNA perciben su trabajo como una aportación valiosa a la familia al contribuir en el ingreso familiar lo cual también beneficia a los menores, prueba de esto fue el observar que en su mayoría el ingreso que recibe el niño por su trabajo es administrado por los padres para la compra de ropa y otras necesidades escolares. Sin embargo, si debe enfatizarse que las familias agrícolas migrantes se encuentran en una situación vulnerable en la que la necesidad de subsistencia obliga a los menores a integrarse en las actividades laborales de los padres/tutores. Si bien esto no excusa la prevalencia del trabajo infantil y la vulnerabilidad a la salud y la integridad física y mental de los NNA, si demuestra un panorama complejo cuya erradicación se vislumbra lejana. Esta erradicación del trabajo infantil puede seguir diferentes enfoques. La 
falta de servicios aumenta los riesgos a los que están expuestos los NNA, especialmente en las regiones agrícolas y rurales. La provisión de servicios básicos como la comunicación digital puede conducir a una reducción de la participación de los NNA que combina el trabajo con la escuela y aumenta la rentabilidad agrícola de los trabajadores (Beuermann, 2015: 988). El trabajo infantil también puede abordarse desde la economía y alteraciones de los campos agrícolas. Las afectaciones y daños en parcelas agrícolas de las zonas rurales conducen a un mayor nivel de trabajo infantil (Beegle et al., 2006: 871), situación que ocurre con frecuencia en México. Independientemente del enfoque aplicado, los resultados de esta investigación permiten argumentar que no se puede lograr un bienestar infantil si las estrategias y los programas sociales destinados a reducir el trabajo infantil no se basan en fomentar el desarrollo de las capacidades humanas centrales. Las políticas públicas actuales buscan un desarrollo rural, pero consideran solo elementos económicos y no consideran la elevada participación de NNA en actividades agrícolas. Es fundamental que las estrategias para la erradicación de esta situación se basen plenamente en el desarrollo de las capacidades humanas. La investigación demuestra que estas capacidades son elementos clave en el desarrollo personal de un menor. El fomento de capacidades permite que los NNA conozcan y entiendan la realidad y favorezcan el proceso de socialización. En consecuencia, se brindarán alternativas de solución para el trabajo infantil y sobre todo se establecerán bases para un crecimiento integral de los NNA para un bienestar y el desarrollo humano en las sociedades de zonas rurales.

\section{REFERENCIAS BIBLIOGRÁFICAS}

APA, 2010, Principios éticos de los psicólogos y código de conducta American Psychological Association (APA) Enmiendas 2010. Recuperado de http://www. psicologia.unam.mx/documentos/pdf/comite_etica/Codigo_APA.pdf

Achenbach, T., 1991, Manual for the Child Behavior Checklist/4-18 and 1991 Profile. Universidad de Vermont: Burlington.

Alacahan, O., 2010, "Socio-economic profiles, attitudes and expectations of families whose children working on street", in Social Policy Conference 59(2):133147. Disponible en https://doi.org/10.1177/097133360902100207

Azaola-Calderon, M.C., 2010, "Importance, meaning and participation within the schooling process of rural areas. An ethnographic study in Michoacán, Mexico", en Perfiles Educativos (32)130:67-82

Baland, J.M. y Robinson, J., 2000, “Is child labor inefficient?”, in Journal of Political Economy 108(4): 663-679. Disponible en http://doi.org/10.1086/316097 
Bienestar infantil y capacidades humanas entre niños, niñas y adolescentes (NNA) ... / F. PÉREZ SOTO et al.

Beegle, K., Dehejia, R. y Gatti, R., 2006, "Child labor and agricultural shocks", in Journal of Development Economics 81(2006):80-96. Disponible en http://doi. org/10.1016/j.jdeveco.2005.05.003

Beegle, K., Dehejia, R. y Gatti, R., 2009, "Why should we care about child labor? The education, labor market, and health consequences of child labor", in The Journal of Human Resources 44(4):871-889. Disponible en https://doi.org/10.3386/ w10980

Berdan, L., Keane, S. y Calkins S., 2009, “Temperament and Externalizing Behavior: Social Preference and Perceived Acceptance as Protective Factors", in Developmental Psychology 44(4):957-968. Disponible en https://doi.org/10.1037/00121649.44.4.957

Beuermann, D., 2015, "Information and communications technology, agricultural profitability and child labor in rural Peru", in Review of Development Economics 19(4):988-1005. Disponible en https://doi.org/10.1111/rode.12180

Bouoiyour, J., y Miftah, A., 2014, "Household welfare, international migration and children time allocation in rural Morocco", in Journal of Economic Development 39(2):75-95.

De Carvalho, F., 2012, "Household income as a determinant of child labor and school enrollment in Brazil: Evidence from a social security reform", in Economic Development and Cultural Change 60(2):399-435. Disponible en https://doi. org/10.1086/662576

Cavalcanti, T., 2003, "Child labor and school policies", in Brazilian Journal of Economics Revista Brasileira de Economia 57(4):741-753

Debebe, Z., 2010, Child labor, agricultural shocks and labor sharing in rural Ethiopia. Recuperado de https://repub.eur.nl/pub/18702/wp491.pdf

Dumas, C., 2012, "Does work impede child learning? The case of Senegal”, in Economic Development and Cultural Change 60(4):773-793. Disponible en https://doi.org/10.1086/665603

Falagas, M.; Matthaiou, D.; Rafailidis, P.; Panos, G. and Pappas G., 2008, "Worldwide prevalence of head lice", in Emerging Infectious Diseases 14(9):1493-1494. Disponible en http://doi.10.3201/eid1409.080368

Ferguson, K., 2005, "Child labour and social capital in the mezzosystem: familyand community-based risk and protective factors for street-working children in Mexico", in Journal of Social Work Research and Evaluation 6(1):101-118.

Gardner, H., 1989, "Multiple intelligences go to school: Educational implications of the theory of multiple intelligences", in Educational Researcher 18(8):4-23. Disponible en https://doi.org/10.3102/0013189X018008004

Gasper, D., 1997, "Sen's capability approach and Nussbaum's capabilities ethic", in Journal of International Development 9(2):281-302. Disponible en https://doi. org/10.1002/(SICI)1099-1328(199703)9:2<281::AID-JID438>3.0.CO;2-K 
Geake, J., 2008; "Neuromythologies in education", in Educational Research. 50(2):123-133. Disponible en https://doi.org/10.1080/00131880802082518

Gilman, L., 2012, The theory of multiple intelligences. Indiana University.

Goodman, D.; Burke, A. and Livingston, E., 2013, "Head Lice", in The Journal of the American Medical Association 309(22):2398. Disponible en http:// doi:10.1001/jama.2013.4430

Hamada-Bassett H., Denham, S., Fettig, N., Curby, T., Mohtasham, M. y Austin N., 2016, "Temperament in the classroom: children low in surgency are more sensitive to teachers' reactions to emotions", in International Journal of Behavioral Development 41(1):4-14. Disponible en https://doi.org/10.1177/0165025416644077

He, H., 2016, "Child labour and academic achievement: Evidence from Gansu Province in China", in China Economic Review 38(2016):130-150. Disponible en https://doi.org/10.1016/j.chieco.2015.12.008

INEGI, 2019, Estadísticas a propósito del día mundial contra el trabajo infantil (datos nacionales). Recuperado de https://www.inegi.org.mx/contenidos/saladeprensa/aproposito/2019/infantil2019_Nal.pdf

Juárez, D. y Rodríguez, C., 2016, "Factores que afectan a la equidad educativa en escuelas rurales de México", en Pensamiento Educativo. Revista de Investigación Educacional Latinoamericana 53(2):1-15. Disponible en https://doi.org/10.7764/ PEL.53.2.2016.8

Miranda, S., 2015, "Caracterización del trabajo infantil rural en México", en Revista Facultad de Ciencias Económicas 27(1): 151-168. Disponible en https://doi. org/10.18359/rfce.3314

Martínez-Villegas F., Galicia-Villanueva S., Domínguez-Galicia Y., Escamilla-García P.E. y Serna-Álvarez H., 2017, "Creation of a Business Innovation Center within a Higher Education Institution as a strategy to develop a culture of innovation among its students", en Navarrete-Cazales Z. y Navarro-Leal M.A. (editores), Innovation in education: Management, curriculum and technologies (pp. 87-99). Plaza and Valdez Editores: Ciudad de México.

Mert, K. y Kadioglu, H., 2014, "The reason why children work on the streets: A sample from Turkey", in Children and Youth Services Review (44):171-180. Disponible en https://doi.org/10.1016/j.childyouth.2014.06.012

Monereo-Atienza, C., 2005, "Tools for a theory of social rights (Doctrinal discussion)", in Yearbook of Philosophy of Law (22):265-290.

Monroy, F. y Juan, J., 2009, “Agricultura y migración campesina. Un estudio para comprender la incorporación del trabajo infantil en una región indígena de México", en Revista Argentina de Sociología 7(12-13): 125-149.

Nussbaum, M. y Sen, A., 1993, The quality of life. Clarendon Press: Oxford.

Nussbaum, M., 1997, "Capabilities and human rights", in Fordham Law Review (66)2:273-300. 
Bienestar infantil y capacidades humanas entre niños, niñas y adolescentes (NNA) ... / F. PÉREZ SOTO et al.

Nussbaum M., 2000, Women and human development: The capabilities approach. Cambridge University Press: Nueva York.

Nussbaum, M., 2011, Creating capabilities: The human development approach. Harvard University Press: Nueva. York.

O’Donnell, O., Doorslaer, E., y Rosati, F., 2005, "Health effects of child work: Evidence from rural Vietnam", in Journal of Population Economics 18(3):437-467. Disponible en https://doi.org/10.1007/s00148-004-0197-y

OIT, 2003, Consideraciones éticas para la investigación sobre NNA y niñas sometidos a las peores formas de trabajo infantil en Nepal. Documento III-02 del MPA PDD: Recuperado de http://www.ilo.org/ipec/Informationresources/ WCMS_IPEC_PUB_11572/lang--es/index.htm

Ordorica, M., Rodriguez, C., Velázquez, B. and Maldonado I., 2009, “The Ethnolinguistic Replacement Index among the Indigenous Population of Mexico", in Desacatos, Journal of Social Sciences 29:123-140. Disponible en https://doi. org/10.29340/29.436

Power, T., 2000, Play and Exploration in Children and Animals. Lawrence Erlbaum Associates Publishers: Londres.

Ramírez-Romero, S., Palacios-Nava, D. y Velazco-Samperio, D., 2006, Diagnosis on the social condition of the children and internal migrants, children of agricultural day laborers. Recuperado de https://www.unicef.org/mexico/spanish/mx_resources_diagnostico_ninos_jornaleros.pdf

Rogers, Carl ,1954, "Toward a theory of creativity", in ETC: A Review of General Semantics (11)4:249-260

Rohner R., 1987, The warmth dimension: Foundations of parental acceptance-rejection theory: New perspectives on family. SAGE Publications: Washington.

Romero-Barquero, C.E. y Saavedra-Guajardo, E., 2016, "Impact of a recreational program in the resilience of $7^{\text {th }}$ grade students at elementary school", in Liberabit - Peruvian Journal of Psychology 22(1):43-56.

Salazar, M., 1996, "El trabajo infantil en América Latina”, en Revista Colombiana de Educación 33(1996): 1-13. https://doi.org/10.17227/01203916.5397

Tang, C., Zhao, L. y Zhao, Z., 2016, "Child labor in China”, in Forschungsinstitut zur Zukunft der Arbeit Institute for the Study of Labor 9976(2016): 1-42. Disponible en https://doi.org/10.1016/j.chieco.2016.05.006

Thabet, A., Matar, S., Carpintero, A., Bankart, J. y Vostanis, P., 2010, "Mental health problems among labour children in the Gaza Strip", in Child: Care, Health and Development (37)1:89-95. Disponible en https://doi.org/10.1111/j.13652214.2010.01122.x

Torres, L., Ortega, L., Garrido A. y Reyes, A., 2008, "Dinámica familiar en familias con hijos e hijas", en Revista Intercontinental de Psicología y Educación 10(2): 31-56.

Sen, A., 1999, Development as freedom. Oxford University Press: Nueva York. 
UNICEF, 1959, Declaration of the rights of the child. The United Nations Children's Fund Recuperado de https://www.unicef.org/malaysia/1959-Declarationof-the-Rights-of-the-Child.pdf

\section{RESUMEN CURRICULAR DE LOS AUTORES}

Francisco Pérez Soto

Doctor en Economía por el Colegio de Postgraduados, profesor-investigador de tiempo completo en la División de Ciencias Económico Administrativas de la Universidad Autónoma Chapingo. Es miembro del Sistema Nacional de Investigadores Nivel 1. Entre sus últimas publicaciones se encuentran las obras: The effect of rainfall on mexican migration to USA, Capacidades humanas y centrales en los jornaleros agricolas migrantes de México, Migration and Remittances on Mexican economic growth, Agricultura, Ciencia y Sociedad Rural, 1810-2010. Volumen II: Agroindustria, Comercio y Mercados. Universidad Autónoma Chapingo. Investigación en matemáticas, economía y ciencias sociales. 2014. Universidad Autónoma Chapingo. Administración de riesgos. Volumen V. Mercados bursátiles y estrategias corporativas. 2014. Universidad Autónoma Metropolitana.

Dirección electrónica: perezsotofco@gmail.com

Registro ORCID: https://orcid.org/0000-0002-7982-420X

Pablo Emilio Escamilla García

Es Maestro en Ciencias en Administración por el Instituto Politécnico Nacional y Doctor en Ingeniería Mecánica por la Universidad de Manchester, Reino Unido. Realizó una estancia posdoctoral en la División de Ciencias Económico-Administrativas de la Universidad Autónoma Chapingo. Fue coordinador de la Maestría en Ciencias en Administración de Negocios en la Escuela Superior de Comercio y Administración unidad Tepepan del IPN. Fue ganador del premio a la Investigación en el IPN en la categoría de investigación desarrollada por investigadores jóvenes. Es miembro del Sistema Nacional de Investigadores y profesor de tiempo completo en el Instituto Politécnico Nacional. Sus últimas publicaciones incluyen: Technical and economic analysis of energy generation from waste incineration in Mexico; Feasibility of energy generation by methane emissions from a landfill in southern Mexico y Eficiencia y confiabilidad de modelos de estimación de biogás en rellenos sanitarios.

Dirección electrónica: peescamilla@ipn.mx

Registro ORCID: https://orcid.org/0000-0001-6704-521X 


\section{Esther Figueroa Hernández}

Doctora en Ciencias en Economía Agrícola por la Universidad Autónoma Chapingo, profesor-Investigador de tiempo completo de la Licenciatura en Economía, Centro Universitario UAEM Texcoco, Universidad Autónoma del Estado de México. Miembro del Sistema Nacional de Investigadores Nivel I. Entre las publicaciones más recientes figuran: Migration and Remittances on Mexican economic growth; Análisis del desempleo, la migración y la pobreza en México; El proceso de asentamiento de la migración México-Estados Unidos, Los precios de café en la producción y las exportaciones a nivel mundial (2019). Libros: Evolución del Mercado Mexicano del Café; La Emigración Internacional, las Remesas y el Desarrollo Económico en México, entre otras.

Dirección electrónica: esther.f.her@gmail.com

Registro ORCID: https://orcid.org/0000-0001-9680-8984

\section{Rebeca A. Pérez Figueroa}

Maestra en Ciencias por el Colegio de Postgraduados y la Universidad de Bristol, candidata a Doctor en Ciencias por la Universidad de Bristol. Entre sus colaboraciones más actuales se encuentran Capacidades humanas y centrales en los jornaleros agrícolas migrantes de México, Los precios de café en la producción y las exportaciones a nivel mundial, y Las principales variables macroeconómicas en el crecimiento de México 1980-2016. Dirección electrónica: rebeca.perezfigueroa@bristol.ac.uk Registro ORCID: https://orcid.org/0000-0002-7634-5385 